\title{
Curcumin - a golden double edged sword!
}

\author{
${ }^{1}$ Dr. Asha M.L., ${ }^{2}$ Dr Aprajita Dua ${ }^{3}$ Dr. Chaitanya Reddy, \\ ${ }^{4}$ Dr. Basetty Neelakantam Rajarathnam, \\ ${ }^{5}$ Dr. Mahesh Kumar H.M, ${ }^{6}$ Dr Lekshmy.J \\ ${ }^{1 .}$ Department of Oral Medicine and Radiology, Dr. Syamala Reddy Dental College, Hospital and Research \\ Centre, Bangalore; MDS. \\ ${ }^{2}$. Department of Oral Medicine and Radiology, Dr. Syamala Reddy Dental College, Hospital and Research \\ Centre, \#111/1, SGR College Main Road, Munnekolala, Marathahalli (Post), Bangalore- 560037; (MDS). \\ 3. Department of Public Health Dentistry,Dr. Syamala Reddy Dental College, Hospital and Research Centre, \\ Bangalore; MDS. \\ ${ }^{4}$.Department of Oral Medicine and Radiology, Dr. Syamala Reddy Dental College, Hospital and Research \\ Centre, Bangalore; $M D S$ \\ ${ }^{5}$ Department of Oral Medicine and Radiology, Dr. Syamala Reddy Dental College, Hospital and Research \\ Centre, Bangalore; $M D S$ \\ 6. Department of Oral Medicine and Radiology, Dr. Syamala Reddy Dental College, Hospital and Research \\ Centre, Bangalore; $M D S$
}

\begin{abstract}
Turmeric (Curcuma longa) is extensively used as a spice, food preservative and colouring material in South East Asia, China and. India. It has been used in traditional medicine as a household remedy for various diseases, including hepatic disorders, biliary disorders, anorexia, cough, diabetic wounds, , rheumatism and sinusitis.

For the last few decades, extensive work has been done to establish the biological activities and pharmacological actions of turmeric and its extracts. Curcumin (diferuloylmethane), which is the main yellow bioactive component of turmeric has shown to have a wide spectrum of biological actions. These include its antioxidant,antiinflammatory, anticarcinogenic antimutagenic, anticoagulant, antifertility, antidiabetic, antibacterial, antifungal, antiprotozoal, antiviral, antifibrotic, antivenom, antiulcer, hypotensive and hypocholesteremic activities.
\end{abstract}

\section{Introduction}

Curcumin, the principal curcuminoid found in turmeric, is considered as its most active constituent. (figure 1)Demethoxycurcumin and bisdemethoxycurcumin are other curcuminoids found in turmeric. It is used as a spice in Indian, Southeast Asian, and Middle Eastern cuisines. In India besides being used for its medicinal purposes for centuries,its extracts are also used as food coloring agents.(1)

The benefits of curcumin in medicine is extremely well known, stretching back to ancient times. Digestive and liver problems, skin diseases, biliary disorders, anorexia, cough, hepatic disorders, bloody urine, hemorrhage, toothache, rheumatism, sinusitis, bruises and wounds are some of the cases where its use has been widely employed.In October, 2010, it was reported that curcumin, when combined with the drug cisplatin, "enhances chemotherapy's suppression of head and neck cancer cell growth." The study, conducted by researchers Eric Srivatsan and Marilene Wong, who have investigated curcumin's anti-cancer properties for six years, builds on previous research demonstrating curcumin's ability to suppress growth of other cancer. Curcumin and turmeric are being studied for their effectiveness against a wide range of other conditions, ranging from arthritis to Alzheimer's disease. $(1,2)$

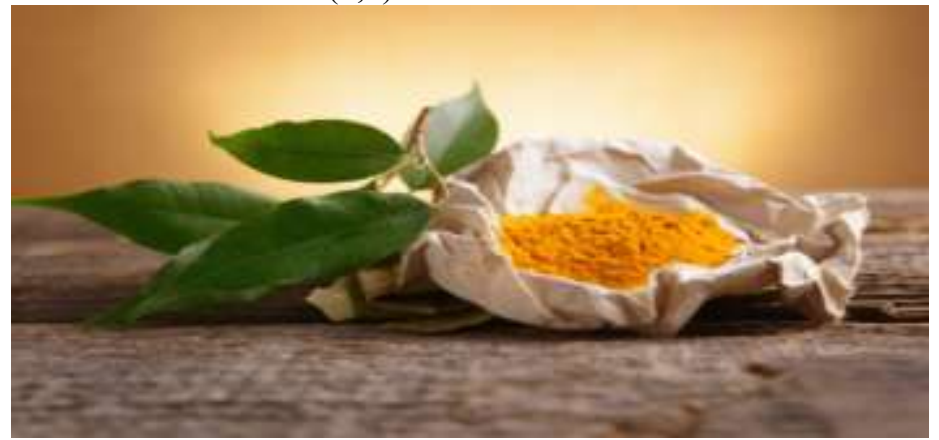

Figure 1 
Turmeric is a spice derived from the of Curcuma longa,a dried ground rhizomes, which belongs to the member of the ginger family (Zingiberaceae). Fat-soluble, polyphenolic pigments known as curcuminoids are mainly responsible for its bright yellow colour.

\section{Chemistry Of Curcumin}

Curcumin incorporates several functional groups. The aromatic ring systems, which are polyphenols are connected by two $\alpha$ - $\alpha$-unsaturated carbonyl groups.

The diketones form stable enols or are easily deprotonated and form enolates, while the $\alpha-\alpha$ unsaturated carbonyl is a good Michael acceptor and undergoes nucleophilic addition. The structure was first identified in 1910. $(1,2)$

\section{Bioavailablity of curcumin:}

The reasons for reduced bioavailability of any agent within the body are poor absorption ,low intrinsic activity, , high rate of metabolism, inactivity of metabolic products and/or rapid elimination and clearance from the body. Efficacy of curcumin as a therapeutic agent for various ailments have been suggested by studies and have a predilection for strong intrinsic actyivity

However, studies over the past three decades related to absorption, distribution, metabolism and excretion of curcumin have revealed poor absorption and rapid metabolism of curcumin that severely curtails its bioavailability. However, the clinical application of curcumin in cancer treatment is considerably limited due to its serious poor delivery characteristics. In order to increase the hydrophilicity and drug delivery capability, it is encapsulated into copolymer. They were much more soluble in water than not only free curcumin but also other biodegradable polymer-encapsulated curcumin nanoparticles. $(3,4)$

\section{Biological activities of curcumin: Antioxidant activity:}

Curcumin is an effective scavenger of both reactive oxygen species and reactive nitrogen species in vitro. It is not clear, however, whether curcumin acts directly as an antioxidant in vivo. Due to its limited oral bioavailability in humans, plasma and tissue curcumin concentrations are likely to be much lower than that of other fat-soluble antioxidants, such as alpha-tocopherol. However, the finding that oral curcumin supplementation ( $3.6 \mathrm{~g} /$ day) for seven days decreased the number of oxidative DNA adducts in malignant colorectal tissue, suggesting that curcumin taken orally may reach sufficient concentrations in the gastrointestinal tract to inhibit oxidative DNA damage., Curcumin may function indirectly as an antioxidant by inhibiting the activity of inflammatory enzymes or by enhancing the synthesis, in addition to direct antioxidant activity of glutathione, an important intracellular antioxidant. $(5,6)$

\section{Anti-inflammatory activity:}

Curcumin has been found to inhibit PLA2, COX-2, and 5-LOX activities in cultured cells. Although curcumin inhibited the catalytic activity of 5-LOX directly, it inhibited PLA2 by preventing its phosphorylation and COX-2 mainly by inhibiting its transcription. $(7,8)$

\section{Inductions Of Cell Cycle Arrest And Apoptosis}

After a cell divides, it passes through a sequence of stages collectively known as the cell cycle before it can divide again. The cell cycle can be transiently arrested to allow for DNA repair or, if the damage cannot be repaired, for activation of pathways leading to cell death (apoptosis) following DNA damage.

The propagation of mutations that contribute to the development of cancer may result in defective cell cycle regulation.(5)

\section{Effects On Autophagic Cell Death}

Autophagy is considered Type II programmed cell death (apoptosis is type I and necrosis is type III) and, thus has come under interest as a potential process that may be exploited in the development of anticancer chemotherapeutics. Curcumin has been shown to be a potent inhibitor of p53.(9)

\section{Inhibition Of Tumor Invasion And Angiogenesis}

Cancerous cells invade normal tissue with the aid of enzymes called matrix metalloproteinases (MMP 9). Curcumin has been found to inhibit the activity of several matrix metalloproteinases in cell culture studies.Invasive tumors must also develop new blood vessels by a process known as angiogenesis, to fuel their 
rapid growth. Angiogenesis has been inhibited by curcumin in cultured vascular endothelial cells and in an animal model. (10)

\section{Disease Prevention \& Treatment Cancer:}

The ability of curcumin to induce apoptosis in cultured cancer cells by several different mechanisms has generated scientific interest in the potential for curcumin to prevent some types of cancer. The inhibition of the development of chemically-induced cancer in animal models of oral, stomach, liver, and colon cancer has been done by the administration of curcumin orally. A supplementation up to $8 \mathrm{~g} / \mathrm{day}$ for three months in patients with precancerous lesions of the mouth (oral leukoplakia), cervix (high grade cervical intraepithelial neoplasia), skin (squamous carcinoma in situ), or stomach (intestinal metaplasia) has been done by a Phase I study at Taiwan.

Histologic improvement on biopsy was intraepithelial neoplasia, two out of six patients with squamous carcinoma in situ, and one out of six patients with intestinal metaplasia. However, cancer developed in one out of seven patients with oral leukoplakia and one out of four patients with cervical intraepithelial neoplasia by the end of the treatment period., Curcumin was shown to "directly and irreversibly" affect the growth of new cancers in experiments on tumors The ability of curcumin to induce apoptosis in a variety of cancer cell lines and its low toxicity has led to scientific interest in its potential for cancer therapy. To date, most of the controlled clinical trials of curcumin supplementation in cancer patients have been Phase I trials. In patients with advanced colorectal cancer found that doses up to $3.6 \mathrm{~g} / \mathrm{day}$ for four months were well-tolerated, although the systemic bioavailability of oral curcumin was low in experiments on tumors.(11)

\section{Inflammatory Diseases}

Although the anti-inflammatory activity of curcumin has been demonstrated in cell culture and animal studies, few controlled clinical trials have examined the efficacy of curcumin in the treatment of inflammatory conditions. A preliminary intervention trial that compared curcumin with a nonsteroidal anti-inflammatory drug (NSAID) in 18 rheumatoid arthritis patients found that improvements in morning stiffness, walking time, and joint swelling after two weeks of curcumin supplementation $(1,200 \mathrm{mg} /$ day $)$ were comparable to those experienced after two weeks of phenylbutazone (NSAID) therapy $(300 \mathrm{mg} /$ day).(11,12)

\section{Dental Application Of Turmeric}

Dental problems: Turmeric can be used in following ways to offer relief from dental problems

- Rinsing the mouth with turmeric water (boil $5 \mathrm{~g}$ of turmeric powder, two cloves, and two dried leaves of guava in $200 \mathrm{~g}$ water) giving instant relief from tooth pain

- Massaging the teeth with roasted, ground turmeric eliminates pain and swelling.

- Applying a paste made from 1 tsp of turmeric with $1 / 2$ tsp of salt and $1 / 2$ tsp of mustard oil provides relief from gingivitis and periodontitis. Rub the teeth and gums with this paste twice daily. (13)

\section{Dental-Plaque Detection System}

Caries or periodontal diseases are thought to be infectious diseases caused by microbes present in dental plaques and it is known that the removal of dental plaques is highly important for the health of oral cavities. However, dental plaques are not easy to identify by the naked eye and it is difficult to confirm their attachment site and extent precisely. Microbial dental plaques are generally stained with dental-plaque staining agents, which contain dyes, to reveal their locations in order to uncover the attached dental plaques.

The dental-plaque detection system includes a dental-plaque staining agent, which contains at least one selected from the yellow pigment of beni-koji, turmeric extracts and curcumin; and a light-emitting apparatus, which outputs light having a wavelength within a range of 250 to $500 \mathrm{~nm}$ to an object in the oral cavity where the dental-plaque staining agent is attached. A yellow pigment of benikoji and turmeric are known as staining agents and also used for other purposes (13)

\section{Pit And Fissure Sealant}

It has been found that tinted pit and fissure sealant is useful for applying to tooth surfaces for the prevention or reduction of dental caries. This sealant can be produced from a composition comprising a polymerizable resin system containing acrylic monomer and at least one colorant selected from the group consisting of Annatto extract, turmeric extract, and â-Apo-8-Carotenal. (13)

\section{Curcumin As A Treatment Modality In Recurrent Aphthous Stomatitis}

Conventional antiseptic gel, the ulcers healed only after the period of time as in previous attacks as shown by many clinical trials in patients. They experienced no early reduction in pain or frequency of recurrence. The 10 patients who used curcumin oil reported that ulcers started healing earlier than in previous 
attacks; there was also early reduction in pain. A follow up for one year has shown no recurrence in these patients. The results of the present study of mucoadhesive semi-solid drug design for the treatment of oral sub mucous fibrosis will be useful for drug industry for the benifit of patients suffering from oral sub mucous fibrosis.(14)

\section{Role Of Curcumin As A Subgingival Irrigant}

Curcumin $1 \%$ as subgingival irrigant resulted in significant reduction in bleeding on probing and redness, when compared with chlorhexidine and saline group as an adjunctive therapy in periodontitis patients. Better results obtained by curcumin irrigation can be attributed to its anti-inflammatory, anti-oxidant properties in resolving inflammation at an earlier stage than chlorhexidine, which act as an anti-bacterial only.(13)

\section{Influence Of Curcumin On Human Gingival Fibroblasts}

Curcumin inhibits the expression of the CCL2 gene, which is also known as monocyte chemo attractant protein-1 (MCP-1). This inhibition was confirmed by Watanabe et al. who showed that (lipopolysaccharide) LPS-induced MCP-1 expression was blocked by curcumin in human gingival fibroblasts,resuting in its apoptosis.(15). This property has been employed in the treatment of oral submucous fibrosis. The total daily dose of $2 \mathrm{~g}$ of Longvida lozenges has been used in the treatment of oral submucous fibrosis for a period of 3 months for resolution of fibrous bands. (16)

\section{Potential Risks And Side Effects}

Extensive in vivo toxicity studies have been performed with turmeric Oleoresin (85\% curcumin) which led to it being placed on the FDA's GRAS (generally recognized as safe) list.. Carcinogenic effects are inferred from interference with thep53tumor suppressor pathway, an important factor inhuman colon cancer. Carcinogenic and LD50 tests inmice and rats, however, have failed to establish a clear relationship between tumorogenesis and administration of curcumin in turmeric oleoresin at above $98 \%$ concentrations. Other in vitro and in vivo studies suggest that curcumin may cause carcinogenic effects under specific conditions. Clinical studies in humans with high doses $(2-12 \mathrm{~g})$ of curcumin have shown few side effects, with some subjects reporting mild nausea or diarrhea. More recently, curcumin was found to alter iron metabolism by chelating iron and suppressing the protein hepcidin, potentially causing iron deficiency in susceptible patients. Further studies seem to be necessary to establish the benefit/risk profile of curcumin. (13)

\section{Conclusion}

The above description prove that curcumin has enormous potential for a variety of diseases. Kawanishiet al.(2005) remarked that curcumin, like many antioxidants, can be a "double-edged sword" where,in the test tube, anticancer and antioxidant effects may be seen in addition to pro-oxidant effects. Well-controlled large clinical trials are required to determine the potential of curcumin both in the prevention and therapy of a disease. All of these studies should further add to the usefulness of curcumin. Overall, the biological safety, combined with its cost and efficacy, and thousands of years of experimentation justify calling curcumin Indian Solid Gold. $(13,15)$

\section{References}

[1]. Curcumin: A wonder anticancer drug. Yogesh Panditrao Palve, P.L. Nayak. Int J Pharm Biomed Sci 2012, 3(2), 60-69.

[2]. Curcumin: The Indian Solid Gold. Bharat B. Aggarwal, Chitra Sundaram, Nikita Malani,and Haruyo Ichikawa SVNY332-Aggarwal December 22, 2006 16:34

[3]. Bioavailability of Curcumin: Problems and Promises Preetha Anand, Ajaikumar B.

[4]. Kunnumakkara, Robert A. Newman, and Bharat B. Aggarwal .Mol. Pharmaceutics, 2007, 4 (6), $807-818$.

[5]. Lechtenberg M, Quandt B, Nahrstedt A. Quantitative determination of curcuminoids in

[6]. Curcuma rhizomes and rapid differentiation of Curcuma domesticaVal and Curcuma

[7]. Sreejayan, Rao MN. Free radicle scavenging activity of curcuminoids. Arneimittelforschy

[8]. 1996; 46(2); 169-171.

[9]. Turmeric and curcumin: Biological actions and medicinal application. Ishita chattapadhya,

[10]. Kaushik Biswas, Udayopathyay and Ranajit Banerjee. Current sciences. Vol 87(1): july 2004.

[11]. Joe B, Vijaykumar M, Lokesh BR. Biological properties of curcumin cellular and molecular mechanisms of action. Crit Rev Food Sci Nutr

[12]. 2004, 44(2), 97-111.[2]

[13]. Sreejayan, Rao MN. Nitrous oxide scavenging by curcumoids. J Pharm 1997;49 (1); 105-107

[14]. Curcumin: A review of anti-cancer properties and therapeutic activity in head and neck squamous cell carcinoma Reason Wilken1, Mysore S Veena1, Marilene B Wan1, Eri S Srivatsan. Molecular Cancer 2011, 10:12.

[15]. Brouet I, Ohshima H. Curcumin, an anti-tumour promoter and antiinflammatory agent, inhibits induction of nitric oxide synthase in activated macrophages. Biochem Biophys Res Commun1995, 206(2),533-540.

[16]. Hazarey VK, Sakrikar AR, Ganvir SM, Efficacy of curcumin in the treatment for oral submucous fibrosis - A randomized clinical trial. J Oral Maxillofac Pathol. 2015 May-Aug;19(2):145-52. 